\title{
Where are we in understanding salamander locomotion: biological and robotic perspectives on kinematics
}

\author{
Konstantinos Karakasiliotis · Nadja Schilling • \\ Jean-Marie Cabelguen · Auke Jan Ijspeert
}

Received: 10 May 2012 / Accepted: 25 November 2012

(C) Springer-Verlag Berlin Heidelberg 2012

\begin{abstract}
Salamanders have captured the interest of biologists and roboticists for decades because of their ability to locomote in different environments and their resemblance to early representatives of tetrapods. In this article, we review biological and robotic studies on the kinematics (i.e., angular profiles of joints) of salamander locomotion aiming at three main goals: (i) to give a clear view of the kinematics, currently available, for each body part of the salamander while moving in different environments (i.e., terrestrial stepping, aquatic stepping, and swimming), (ii) to examine what is the status of our current knowledge and what remains unclear, and (iii) to discuss how much robotics and modeling have already contributed and will potentially contribute in the future to such studies.
\end{abstract}

This article forms part of a special issue of Biological Cybernetics entitled "Lamprey, Salamander Robots and Central Nervous System".

K. Karakasiliotis $(\varangle)$. A. J. Ijspeert

École Polytechnique Fédérale de Lausanne (EPFL), Lausanne,

Switzerland

e-mail: kostas.karakasiliotis@epfl.ch

A. J. Ijspeert

e-mail: auke.ijspeert@epfl.ch

N. Schilling

Institute of Systematic Zoology and Evolutionary Biology,

Friedrich-Schiller-University, Jena, Germany

e-mail: nadja.schilling@tiho-hannover.de

N. Schilling

University of Veterinary Medicine Hannover, Foundation Small

Animal Clinic, Hannover, Germany

J.-M. Cabelguen

Neurocentre Magendie INSERM U862, Bordeaux University, Bordeaux-cedex, France

e-mail: jean-marie.cabelguen@inserm.fr
Keywords Salamander - Kinematics · Walking · Swimming $\cdot$ Salamander-like robots

\section{Introduction}

For almost a century, researchers from various fields have used salamanders to study amphibious tetrapod locomotion. Particularly, evolutionary biologists interested in one of the key events in vertebrate evolution, the transition from water to land, owe much of their current understanding of early tetrapod locomotion to the salamander. This is illustrated by the long list of publications suggesting that salamanders resemble early representatives of tetrapods in their skeletal morphology and locomotor mode more than any other extant species (Schaeffer 1941; Carroll 1988; Worthington and Wake 1972; Romer and Byrne 1931; Howell 1944; Barclay 1946; Gray 1968; Edwards 1989; Gao and Shubin 2001; Clack 2002a). It has been suggested that the sprawling posture (Gregory and Camp 1918; Romer 1922; Rewcastle 1981; Ashley-Ross 1994a,b; Ashley-Ross and Lauder 1997; Ashley-Ross and Bechtel 2004; Ashley-Ross et al. 2009) as well as the undulatory movements of the spine (Daan and Belterman 1968; Frolich and Biewener 1992; D'Août and Aerts 1997) of modern salamanders resemble those of early tetrapods. The salamander has also attracted the interest of neuroscientists in the neuronal mechanisms that drive the various modes of locomotion in salamanders (Chevallier et al. 2008; Cabelguen et al. 2010).

More recently, salamanders have captured the interest of both computational neuroscientists and biomimetic roboticists. Their amphibious lifestyle requires clear changes in their kinematics, which makes salamanders good study objects for flexible control mechanisms and substrate adaptation strategies (e.g., stepping vs. swimming). Although the 
sprawling posture is considered to be intrinsically unstable by biologists owing to the inability to balance the center of gravity over the limbs (Ashley-Ross 1994a; Hildebrand 1966, 1985; Gray 1968; Edwards 1977), for robotics, it can be argued that this posture can prevent a robot from toppling and therefore can be considered as rather stable. Moreover, Gray (1944) stated that, despite their intrinsic disadvantage, the gait most commonly observed in salamanders, the walking trot, is the most stable among tetrapod gaits.

Engineering has always stimulated locomotor studies and developed tools to allow new insights and make modeling faster and more precise. Cinematography has been used by many investigators early on (e.g., Gray 1944; Evans 1946) to study the kinematics of salamanders. Force-plate measurements (Barclay 1946; Sheffield and Blob 2011) and electromyography (e.g., Székely et al. 1969; Frolich and Biewener 1992; Ashley-Ross 1995; Delvolvé et al. 1997; Deban and Schilling 2009) helped to understand their dynamics and muscular activity patterns. Using cineradiography, we were recently able to record and reconstruct the kinematics of the salamander's skeleton in great detail (Karakasiliotis et al., under preparation). Furthermore, robots and neuromechanical models facilitated the study of the neural mechanisms underlying gait generation in salamanders (Ijspeert et al. 2007; Ijspeert 2008).

The goal of this article is to review our current understanding on the salamander's kinematics. For this, we first recap previous studies and complement and compare the results with our recent findings from the detailed kinematic analysis of Pleurodeles waltl. Using our X-ray recordings, we are able to reconstruct the three dimensional kinematics of the salamander and present averaged motion patterns for most skeletal elements. We will then review the studies that used numerical simulation or robots to explore the kinematics of salamander-like models and will discuss the potential of such approaches in exploring animal locomotion.

\section{The salamander's morphology}

To characterize the kinematics of an animal and to postulate general gait patterns, it is important to first evaluate interspecific morphological variability and to which extend it may affect locomotion.

Salamanders appear in the fossil record around 150 million years ago (Gao and Shubin 2001). Some extant species (of the approximately 550 species in the order Caudata) are well adapted to the aquatic environment, while others spend most of their time in terrestrial habitats. Differences in the tail morphology have been reported for example for species preferring these different habitats (Worthington and Wake 1972).
Independent from interspecific variations, the typical salamander skeleton consists of four main components: (i) the skull, (ii) the elongated spine with a cervical, trunk, sacral, and tail region, (iii) the forelimbs, and (iv) the hindlimbs (Fig. 1). The spine consists of serial elements, the vertebrae, interconnected in a way to allow first and foremost lateral bending. Overall, fore- and hindlimbs possess three serially homolog segments (from proximal to distal): the humerus/femur, ulna and radius/tibia and fibula, arranged as parallel bones, and the feet. One difference between the fore- and the hindlimbs is the number of digits, which is four and five, respectively. A second difference is the connection between the legs and the spine. The pelvic girdle is directly connected with the spine at the sacrum, while the pectoral girdle has no skeletal connection to the spine and can move independently from it.

A distinctive example of morphological variation is Siren lacertina, which lacks hindlimbs and its whole-body kinematics differ therefore significantly from four-legged species (Azizi and Horton 2004). Simons and Brainerd (1999) reported substantial variation in the hypaxial musculature of different species and discussed its effect on locomotion. Daan and Belterman (1968) reported that the more the reduced the limbs, the slower the speed of movement at which traveling waves appear in the spine. Until now, many of the questions addressing interspecific variability have not been systematically answered because quantitative kinematics for both terrestrial and aquatic locomotion are sparse with only few species studied in more detail (Ashley-Ross et al. 2009). Some studies investigated the effect of body size differences. Bennett et al. (1989), for example, showed that larger individuals are faster and have higher endurance than their smaller conspecifics.

\section{The kinematics of the salamander}

Salamanders demonstrate a rich repertoire of gaits and several of them have been previously studied in more detail (i.e., forward terrestrial stepping, backward stepping, underwater stepping, and swimming). In this section, we give an overview of the studied gaits along with the current knowledge of the spinal and limb kinematics for each of these gaits.

\subsection{Terrestrial stepping}

\subsubsection{Gaits}

Forward terrestrial stepping On land, salamanders use coordinated motions of the flexible spine and the limbs to propel their body forward. Forward terrestrial stepping has been investigated by many researchers (Gray 1944, 1968; Barclay 1946; Evans 1946; Roos 1964; Daan and Belterman 

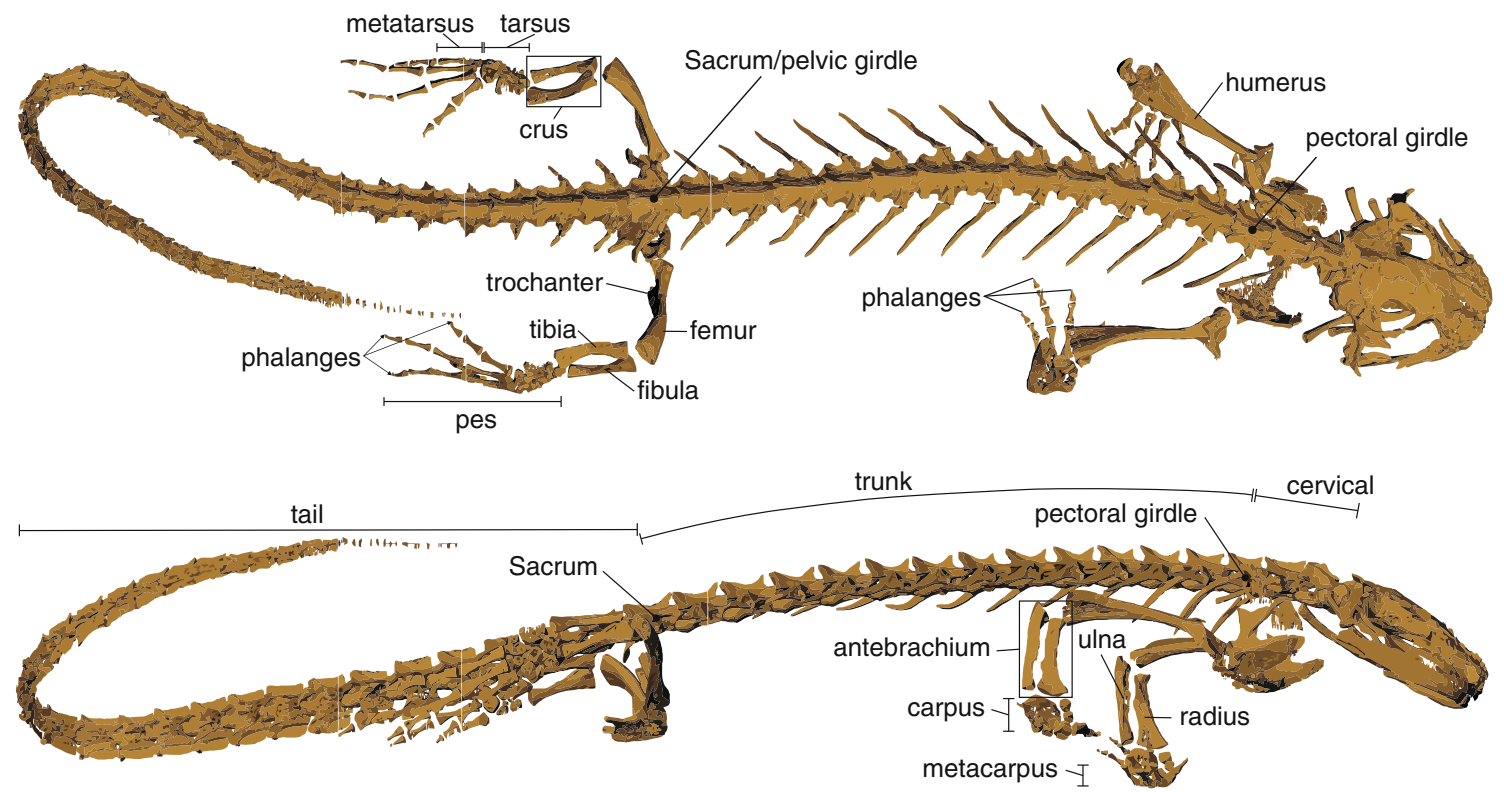

Fig. 1 The skeleton of the salamander Pleurodeles waltl reconstructed from CT scans illustrating the general skeletal morphology of a four-legged salamander from the dorsal and the lateral perspectives

1968; Hildebrand 1976, 1985; Edwards 1977, 1989; Peters and Goslow 2010; Gans and De Gueldre 1992; AshleyRoss 1994a,b; Ashley-Ross et al. 2009). Hildebrand (1976) classified the slow walking gait of salamanders as a lateral sequence walk and fast walking as a trot. In a lateral sequence, the footfall of a hindfoot is followed by the touchdown of the ipsilateral forefoot, then the contralateral hindfoot and finally the contralateral forefoot make ground contact. In a trot, the diagonal limbs move more or less in synchrony (Fig. 2). Some variations of the typical lateral sequence walk were reported by Ashley-Ross (1994a,b). In these variations, the footfalls of the ipsilateral fore- and the contralateral hindfoot were reversed, and the hindlimb completed its swing phase before the diagonal forelimb.

Edwards (1977) contributed significant experimental and theoretical insight on the kinematics of forward terrestrial stepping by studying 48 species of salamanders. Although quantitative details were not included, he reported a considerable overlap between the speed ranges of the lateral sequence walk $(0.1-3.16 \mathrm{BL} / \mathrm{s}, \mathrm{BL}=$ body length) and the trot $(0.15-4.78 \mathrm{BL} / \mathrm{s})$. He also reported very fast gaits during which the salamanders seemed to almost swim on the surface much like the behavior observed earlier by Evans (1946). However, both authors noted that these gaits were escape responses and not sustainable. In the same work, Edwards estimated the contribution of the three means by which body propulsion can be achieved during walking (Gray 1968; see also Peters and Goslow 2010; Ashley-Ross 1994a, p. 273 for discussions): (i) girdle rotation, i.e., propulsion is generated by bending the vertebral column and thereby rotating the limb girdles, (ii) limb retraction, i.e., propulsion is generated only by limb retraction while the trunk is straight, and (iii) humeral and femoral rotation, i.e., propulsion is generated by swinging the lower limbs back and forth due to humeral and femoral long-axis rotation. Edwards (1977) concluded that salamanders use all three means during locomotion and estimated that limb retraction contributes 56-62\%, limb rotation 26-28\% and girdle rotation 10-18\% to body propulsion. Contrary to Roos (1964), and in agreement with Evans (1946) and Ashley-Ross and Lauder (1997), Edwards stressed the fact that all species walked with their ventral aspect of the trunk cleared of the ground. However, this was not the case for Pleurodeles waltl (see bending in Sect. 3.1.2). In terms of quantitative kinematics, a large body of work has been presented by Ashley-Ross et al., including detailed two-dimensional (Ashley-Ross 1994a) and threedimensional (Ashley-Ross et al. 2009) kinematics of forward terrestrial stepping.

Backward terrestrial stepping The first kinematic details on backward terrestrial stepping in salamanders were provided by Ashley-Ross and Lauder (1997). In this mode, the salamanders did not show one of the previously classified gaits (Hildebrand 1976; Fig. 2) and their ventral aspect of the trunk was not always lifted from the ground (in contrast to forward walking). The sequence of footfalls was: left hindlimb, right forelimb, left forelimb, and right hindlimb. Mean stance duration was longer (79\%) compared to forward walking $(67 \%)$, which may be related to the much slower walking speed during backward walking $(0.13 \mathrm{SVL} / \mathrm{s}$ vs. $0.75 \mathrm{SVL} / \mathrm{s}, \mathrm{SVL}=$ snout vent length). Moreover, during 

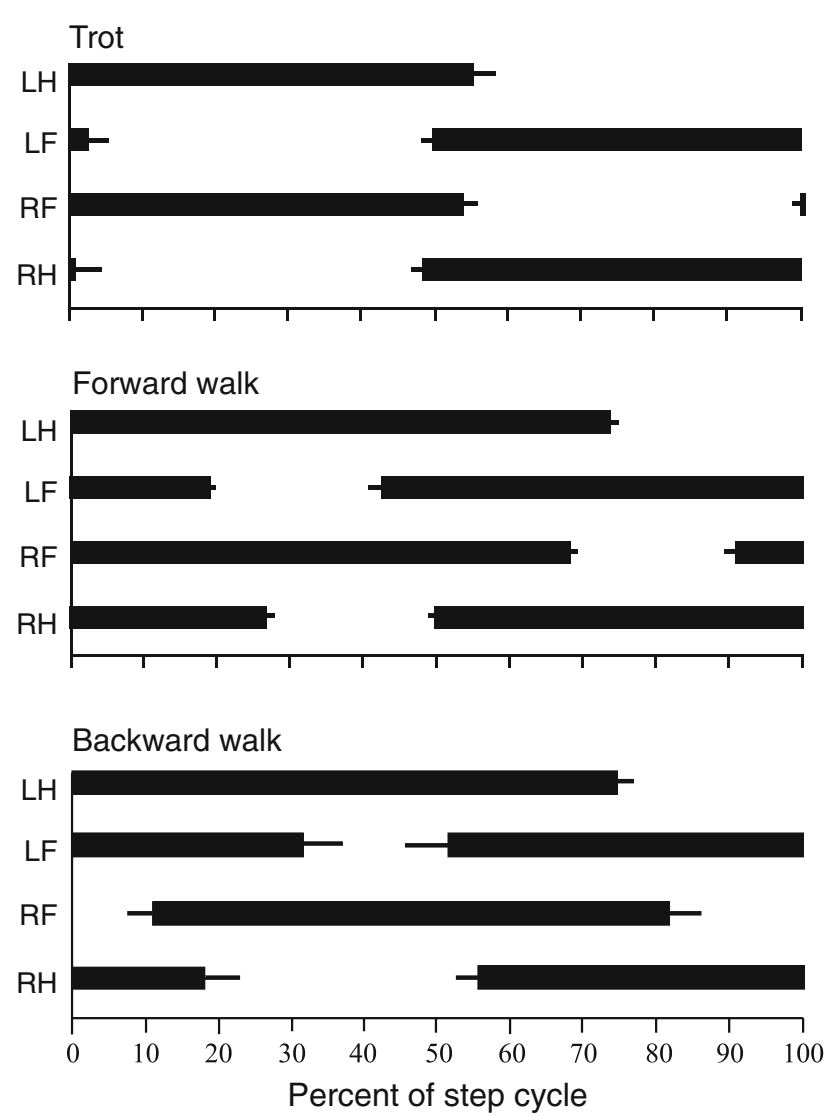

Fig. 2 Representative mean gait diagrams of salamanders during trotting, forward walking and backward walking. The error bars denote the standard deviation for multiple locomotor cycles of the same species of salamanders (data extracted from Ashley-Ross 1994a; Ashley-Ross and Lauder 1997)

backward walking, the toes are the first to make ground contact and stance phase ends with heel off. In forward walking, however, Evans (1946) showed by his kymographic recordings that only the toes were in contact with the ground at any time, while the heel never touched the substrate.

\subsubsection{Spinal kinematics during terrestrial stepping}

Edwards (1977) estimated that girdle rotation contributes $26-28 \%$ to body propulsion. Robotic studies have confirmed that body bending and therefore girdle rotation significantly increases locomotor speed (see Sect. 4.2; Fig. 9). Therefore, lateral undulations of the trunk play an important role in walking salamanders. Gray (1968) and Edwards (1977) argued that good coordination between trunk bending and limb footfalls can increase stride length without any further contribution by limb retraction (Fig. 1 in Edwards 1977). That proper coordination between trunk bending and limb-footfalls can increase stride length was also shown in robotic studies (see Sect. 4.2; Fig. 11). Furthermore, it was shown that there is only a small range of trunk-limb phase difference at which forward speed is not significantly affected, while bad coordination yielded much slower or even no forward propulsion.

Spinal kinematics in salamanders can be described as bending and twisting motions. Thereby, lateral bending occurs in the horizontal and dorsoventral bending in the sagittal plane, while twisting refers to counterclockwise rotations about the long-axis of the body.

Lateral bending One of the earliest observations on salamander locomotion concerned the different types of spinal undulations. The main debate among authors has been about whether salamanders use standing or traveling waves. Standing waves occur when the positions of maximal lateral excursion remain stationary, forming $\mathrm{C}$-shaped curves of the spine with nodes near the limb girdles. Traveling waves form when maximal excursion propagates along the animal's body axis.

Roos (1964) developed a method to accurately characterize lateral bending at different positions along the spine. In his method, Roos used small consecutive segments along the spine and determined the angle at each intersegmental joint (i.e., he characterized local curvature). Daan and Belterman (1968) extended Roos' isoskolies by connecting the points of maximal bending to the so-called shift line. Edwards (1977), later on, used the same method in his studies.

Several authors stated that salamanders use standing waves when walking (Roos 1964; Daan and Belterman 1968; Frolich and Biewener 1992). More precisely, Frolich and Biewener observed two nodes near the limb girdles, around which the standing waves alternate bending sides. Daan and Belterman reported a tendency toward a traveling wave in the posterior trunk. However, Edwards (1977) questioned such generalization because Roos had filmed only one species, while he studied 48 species and consistently observed standing waves during slow walking and traveling waves during trotting. A traveling wave was also reported by Ashley-Ross (1994a), when the salamanders trotted, while standing waves occurred during walking. It is worth noting that in her discussion, Ashley-Ross states that the traveling waves can be more clearly identified using a stick-figure illustration, in which all trunk points pass along a similar trajectory in space (Fig. 6C and D in Ashley-Ross 1994a). However, if in this plot for each instance a line is drawn between the two extreme points (i.e., the girdles), the trunk will always form a $\mathrm{C}$-shaped curve which gradually changes curvature, becomes straight and then switches bending side. According to us, the latter more closely matches the definition of a standing wave. The traveling-like wave observed from the time lapse of the stick figures and, therefore, the plot of lateral displacement, might most probably be an effect of the welltimed and pronounced changes in the orientation of the trunk with respect to the animal's line of locomotion. This is partially supported by Ashley-Ross, who observed a standing wave when analyzing the lateral trunk flexion, but a traveling wave based on the lateral displacement from the stick 
figures for the same trotting recordings (Fig. 8 in Ashley-Ross 1994a).

Lateral displacement plots have also been used by others to distinguish standing from traveling waves (e.g., Frolich and Biewener 1992). However, as in the example above, it may not be apparent that propagation of maximal lateral displacement means propagation of lateral flexion. Body orientation may be misleading in some cases. This is particularly important for terrestrial locomotion where the direction of locomotion is not directly coupled with the movements of the body (as it is in swimming), but with the limbs. We therefore believe that Roos' method is the most appropriate for characterizing waves of lateral bending because it pays heed to the internal body kinematics independently from the direction of locomotion.

In our cineradiographic recordings, we were able to characterize the lateral bending that occurred during forward terrestrial stepping in two individuals ( $N=23$ cycles total). In particular, we measured the intervertebral angles between all vertebrae up to $1.68 \mathrm{SVL}$. In contrast to most previous studies, the species used in our experiments, Pleurodeles walt, is primarily aquatic. However, the animals walked on a flat substrate at relatively low, self-determined speeds $(0.49 \pm 0.12 \mathrm{SVL} / \mathrm{s}$; cycle duration $=1.52 \pm 0.32 \mathrm{~s})$. In all trials, the animals used a lateral sequence walk (Fig. 3, top) with relatively long stance phases $(\approx 77 \%)$. The ventral aspect of the pectoral girdle had often contact with the substrate which contradicts Edwards' observation (Edwards 1977) for all the species he studied.

Our results confirm a standing wave of spinal motions during walking. Figure 3 presents the results for lateral bending. In particular, in the posterior trunk $\left(J_{9}-J_{15}\right)$ and anterior tail $\left(J_{19}-J_{26}\right)$, the standing waves are almost perfect (i.e., with almost no phase lag between consecutive segments) and these two main standing waves are in exact anti-phase. In accordance with Daan and Belterman (1968), some influence of a traveling wave (i.e., with non-zero phase lags) was found, but in contrast to their observation, a traveling wave occurred in in the anterior trunk $\left(J_{4}-J_{8}\right)$ and the posterior tail $\left(J_{27}-J_{35}\right)$ in Pleurodeles waltl. However, the amplitude in the anterior trunk joints is too small to be unequivocally interpreted as a traveling wave. The curvature of lateral flexion (see joint amplitude on the left in Fig. 3) is maximal on either side of the pelvic girdle and gradually decreases toward the anterior trunk and the posterior tail. It is interesting, albeit functionally unclear, that amplitudes of lateral flexion are nearly symmetrical cranial and caudal to the first two postsacral vertebrae $\left(J_{17,18}\right)$. Ashley-Ross $(1994 a)$ attributed the decreasing amplitude of lateral oscillation in the anterior trunk to the salamander's attempt to keep the head's orientation stable throughout the stride.

Dorsoventral bending Subsequently, there no information in the literature about dorsoventral movements of the sala- mander spine. This is likely due to the sprawling posture being associated with lateral bending and the zygapophyses (lateral appendages at each vertebra) being oriented horizontally in salamanders (Francis 1934). Indeed, in our study, we did not find any oscillatory pattern in sagittal body plane. We measured the dorsoventral bending at four locations along the spine: anterior and posterior trunk and at two positions on the anterior tail. No oscillatory patterns were found and only constant bending was recorded (see amplitude excursions in Fig. 4).

Twisting It has been hypothesized that, during terrestrial locomotion, animals with sprawled limb postures experience long-axis torsion due to the ground reaction forces acting during diagonal limb support (Carrier 1993). Studies testing this hypothesis used electromyography to monitor the activity of the lateral hypaxial musculature (Carrier 1993; Bennett et al. 2001). Quantitative kinematics of long-axis twisting were not available up to our observations from the cineradiographic recordings which will be reported in more detail in Karakasiliotis et al. (under preparation). We characterized the twisting movements of the trunk using the three-dimensional positions of the pectoral and pelvic girdles. Twisting angle was then measured between the lines that connect the hips and shoulders, respectively. Twist excursions appeared to be significant $\left(\approx 10^{\circ}\right.$ amplitude; Fig. 5$)$ and resulted mainly from the long-axis roll of the pelvic girdle, while the pectoral girdle showed very little roll. We hypothesize that the plateau in the twisting angle may reflect maximum possible twist of the trunk due to mechanical constraints, such as the orientation of the zygapophyses, muscular maximal stress, and/or muscular stabilization.

\subsubsection{Hind-limb kinematics during terrestrial stepping}

The skeletal structure of the salamander's tarsus is considered to be the closest available analogue to that of early tetrapods (Schaeffer 1941; Ashley-Ross 1994a). The observed forward orientation of the salamander pes during stepping is also considered to be a primitive feature deduced from fossil trackways (Romer and Byrne 1931; Schaeffer 1941; Niedzwiedzki et al. 2010). Moreover, Edwards (1977) estimated that limb retraction supplies 56-62\% of forward propulsion and limb rotation 10-18\% highlighting the importance and bifunctionality of the limbs. Theoretical considerations and descriptions dealt with the movements and mechanics of the hindlimbs (Barclay 1946; Gray 1968; Edwards 1977, 1989; Peters and Goslow 2010) as well as the limb's musculature and activation patterns in relation to the kinematics (Ashley-Ross 1992, 1995; Delvolvé et al. 1997).

A significant amount of work on quantitative kinematics of the salamander's hindlimbs and their relative timing with trunk bending has been contributed by Ashley-Ross et al. (Ashley-Ross 1994a,b; Ashley-Ross et al. 2009). Most of 
Fig. 3 Characterization of the lateral body oscillations of Pleurodeles waltl (two animals, $N=23$ total). Top footfall pattern. Middle black curves show the oscillation of each intervertebral joint from $J_{1}$ (neck) to $J_{35}(1.68 \mathrm{SVL})$ during forward terrestrial stepping, the gray regions denote their standard deviation. For positive values, the joint is concave with respect to the reference hindlimb. Each circle denotes the zero-crossing of each joint and the horizontal bars around them its standard deviation. The curve that connects these circles is similar to the shift line used by Daan and Belterman (1968). Left Mean values of joint amplitude is shown for each joint with the standard deviation in parenthesis. The horizontal gray bars give a visual representation of the variation of joint amplitude along the body. Right Mean values of phase lag between consecutive joints and their standard deviation in parenthesis. The gray bars give a visual representation of the level of the phase lag, while the sign denotes the direction of the phase difference. Note that each phase lag bar is placed between the respective joints along the vertical axis

Fig. 4 Dorsoventral spinal oscillations of Pleurodeles walt (two animals, $N=23$ total). The figure follows the same conventions as in Fig. 3. The dorsoventral oscillations are shown for four positions along the animal's spine: anterior and posterior trunk and at two positions on the anterior tail as angles projected to the sagittal plane. Anterior trunk is measured as the angle between the vertebrae 1 (atlas), 7, and 11 on the sagittal plane. Posterior trunk represents the angle between the vertebrae 7,11 , and 16 (sacrum). Anterior tail 1 denotes the angle between vertebrae 16 (sacrum), 20, and 25 and anterior tail 2 the angle between vertebrae 20,25, and 32(1.68 SVL). Dorsoventral angles are zero when their segments are in line. Positive values yield upward bending
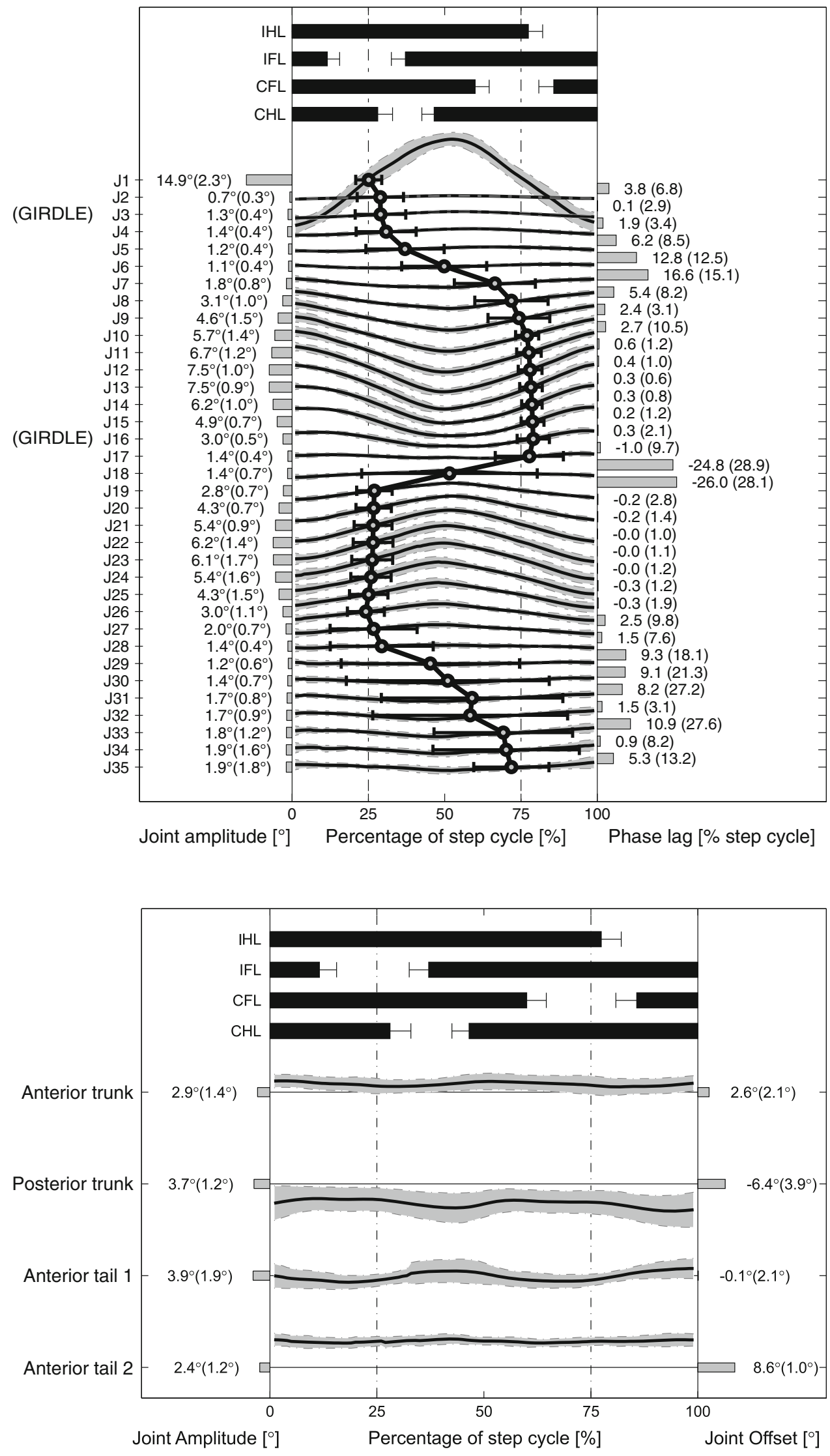


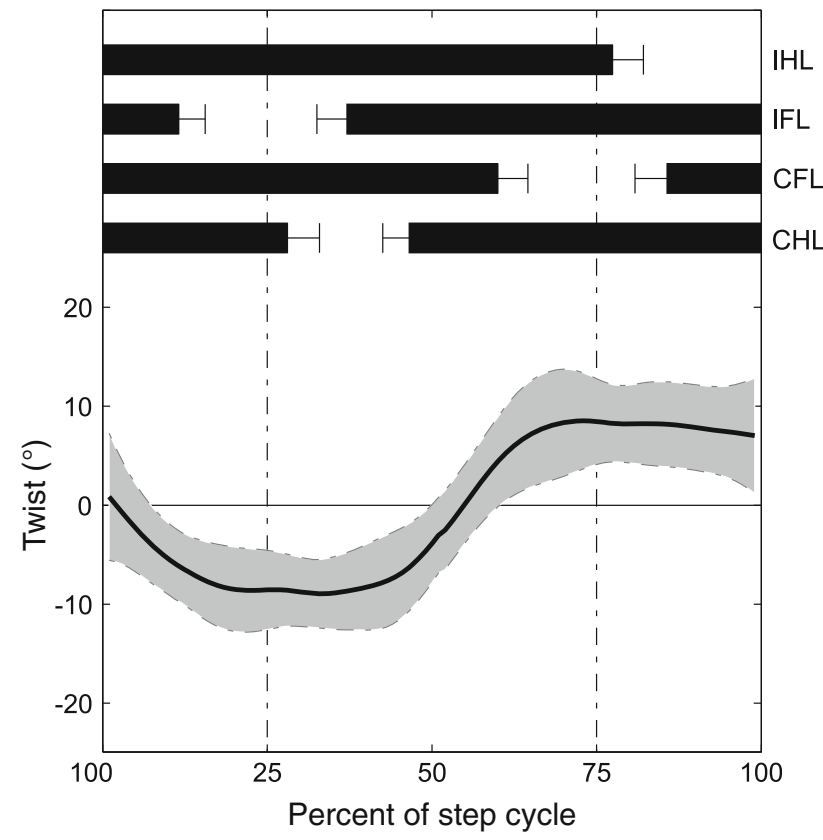

Fig. 5 Twisting angle of the trunk (mean and SD) during terrestrial stepping (two animals, $N=23$ total). Positive values yield clock-wise twisting (right-hand rule). Zero means that shoulders and hips are on the same plane

the variables were described in two dimensions; however, some three-dimensional data were presented in Ashley-Ross et al. (2009). Three-dimensional angles of the hindlimbs were also measured using the skeletal kinematics from our cineradiographic recordings. In addition to the commonly measured variables (i.e., femoral protraction-retraction, femoral abduction-adduction and knee flexion-extension), we calculated the dorsoventral and twist angles of the ankle joint and the twist at the metatarso-phalangeal joint (see caption in Fig. 6 for definitions). Additionally, to decouple rolling movements of the hindlimb from the conventional definitions of protraction-retraction, we deduced individual angular movements at each of the three axes of the hip joint using inverse kinematics techniques. More precisely, we calculated the roll, pitch (up-down femoral movements when roll is zero), and yaw (back and forth femoral movements when roll is zero) angles at the hip joint. ${ }^{1}$ Unfortunately, the majority of the parameters of the two data sets collected by AshleyRoss and from cineradiography cannot be directly compared. As the angular kinematics derived from the cineradiographic experiments are all in three dimensions, we will mainly

\footnotetext{
${ }_{1}^{1}$ Roll, pitch and yaw are the angles which describe the rotation around each one of the three axes of a joint and should not be confused with retraction and adduction. Retraction or adduction can be achieved with different combinations of roll, pitch and yaw. The sequence is also important. For example, when roll is zero, pitch can lead to adduction/abduction only. In any other case, it will cause some retraction/protraction.
}

use the plots from Fig. 6 to describe the limb movements. Whenever possible, observations from Ashley-Ross will be incorporated.

The main role of the hindlimb during locomotion starts with the ground contact of the distal part of the pes. The tarsus is never in contact with the ground during stance phase (Evans 1946). This is supported by our cineradiographic recordings which show that the minimum angle between the tarsus and the substrate was $\approx 20^{\circ}\left(0^{\circ}\right.$ would mean that they are parallel). The initiation of the hindlimb's stance phase coincides with the maximum lateral bending of the trunk to the ipsilateral side (Ashley-Ross 1994a; Fig.3). At this point, the femur is already in retraction (i.e., two-thirds of the swing phase) and maximally adducted (Fig. 6A, B). Throughout stance phase, both femoral retraction and abduction follow almost perfect linear profiles. Retraction, however, decelerates during the swing phase of the contralateral hindlimb and then resumes at its initial pace right after the contralateral hindlimb made ground contact. During the final part of stance, while the toes roll off the ground, retraction has ceased, and the femur remains in its fully retracted position (Fig. 4B in Ashley-Ross 1994a; Fig.6A). Protraction starts instantly after the last toe left the ground. Because maximum retraction is only slightly greater than protraction, the femur moves almost symmetrically around the hip (Table 1 in Ashley-Ross 1994a; Fig.6A). The knee is already in flexion when the hindlimb touches the ground, i.e., the beginnings of knee flexion and femoral retraction are synchronized (two-thirds of swing phase, $\approx 90 \%$ of step cycle; Fig. 6C). During the first third of the stance phase, the knee flexes approximately until the femur is almost perpendicular to the spine $\left(84^{\circ}\right.$, Fig. $\left.6 \mathrm{~A}\right)$ and parallel to the ground $\left(0^{\circ}\right.$, Fig. $\left.6 \mathrm{~B}\right)$. When the trunk starts bending to the contralateral side, the knee begins to extend until the crus is almost aligned with the femur $\left(155^{\circ}\right.$, Fig. $\left.6 \mathrm{C}\right)$. Again, as the maximally retracted femur waits for the toes to leave the ground, the knee maintains its maximally extended position. With the initiation of the swing phase, the knee rapidly flexes while the femur is protracted. When the femur is almost perpendicular to the spine, the knee starts to extend in order to prepare for the next stance.

The crus and pes also show interesting patterns during the stance phase. As with the hip and knee joints, the ankle joint initiates flexion in preparation of the subsequent stance phase almost midway through swing. The ankle, shortly after the femur has become perpendicular to the spine and the crus perpendicular to the ground, extends until the tarsus becomes almost perpendicular to the crus (30\% of step cycle; Fig. 6D). Then, the ankle joint flexes until tarsus and crus are almost aligned. Temporally, this ankle flexion follows the retraction of the femur and the extension of the knee $\left(\approx 160^{\circ}\right)$. The toes are pointing backward at the end of the stance. During swing, the orientation of the toes most likely grad- 


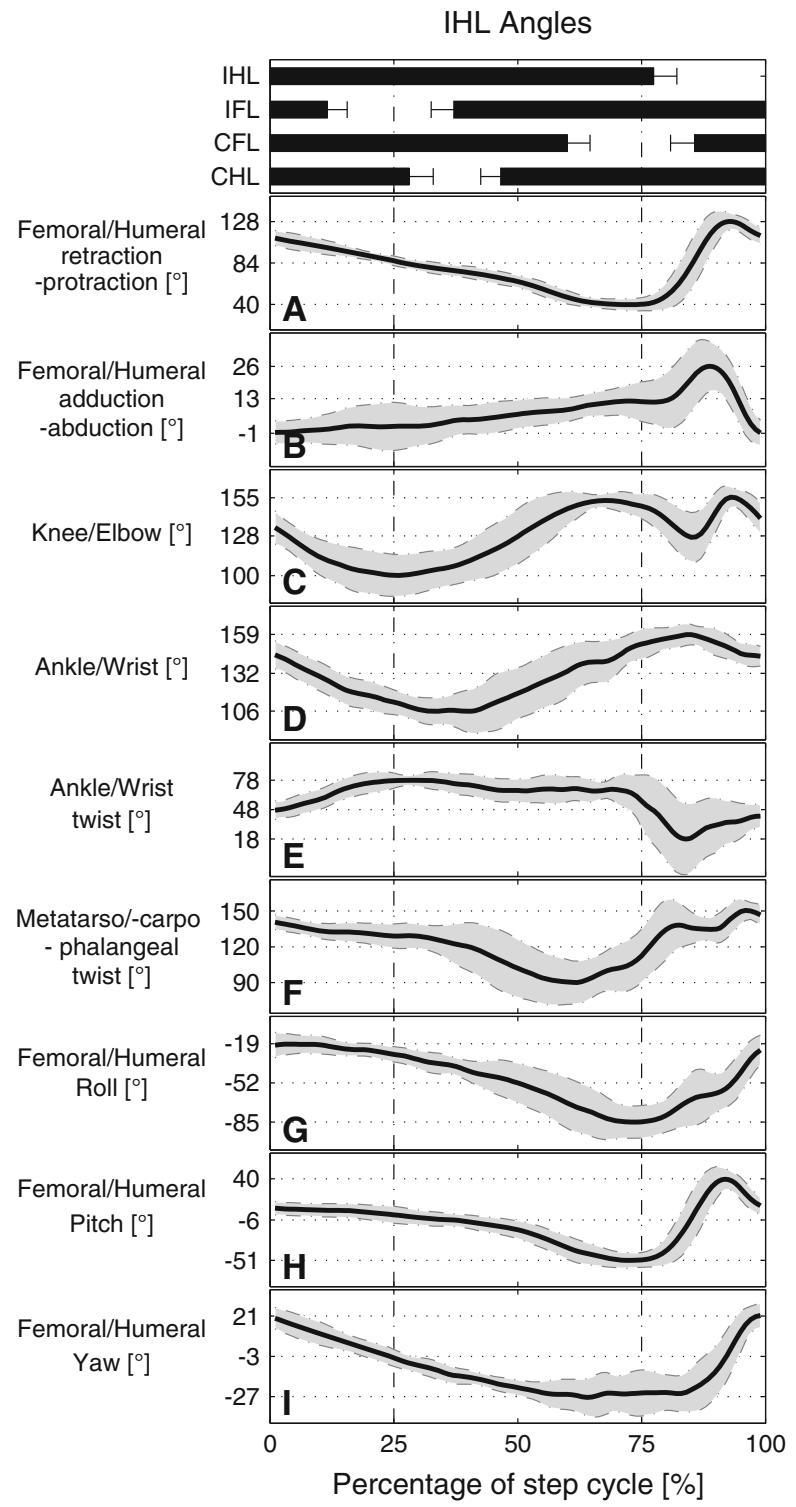

Fig. 6 Angular kinematics of the ipsilateral hindlimb (left column IHL) and the contralateral forelimb (right column CFL). Top the mean footfall patterns are shown with black horizontal bars; their ends representing the means for the time of touch down and lift off, respectively. In each plot, the black line represents the mean value of the angle throughout a stride cycle, the gray area indicates the SD (two animals, $N=23$ total). The highest and lowest values on the $y$-axis represent the maximum and minimum mean values of the corresponding angle, respectively. The following definitions have been used for each kinematic variable. $\mathbf{A}, \mathbf{J}$ The angle between the femur/humerus and the scapula/three vertebrae around the pelvic girdle from the retraction side $\left(90^{\circ}\right.$ is perpendicular to the spine or scapula). $\mathbf{B}, \mathbf{K}$ The adduction-abduction

ually changes to the forward one by a combination of rapid counter-clock-wise (forwards) rotation of the limb (Fig. 6G) and an extension of the ankle joint resulting in lifting the toes up. The foot starts to spread when the ankle joint starts to extend. The foot remains parallel to the line of body progression, supposedly due to the long-axis rotation of the bones of the crus, with the tibia crossing in front of the fibula

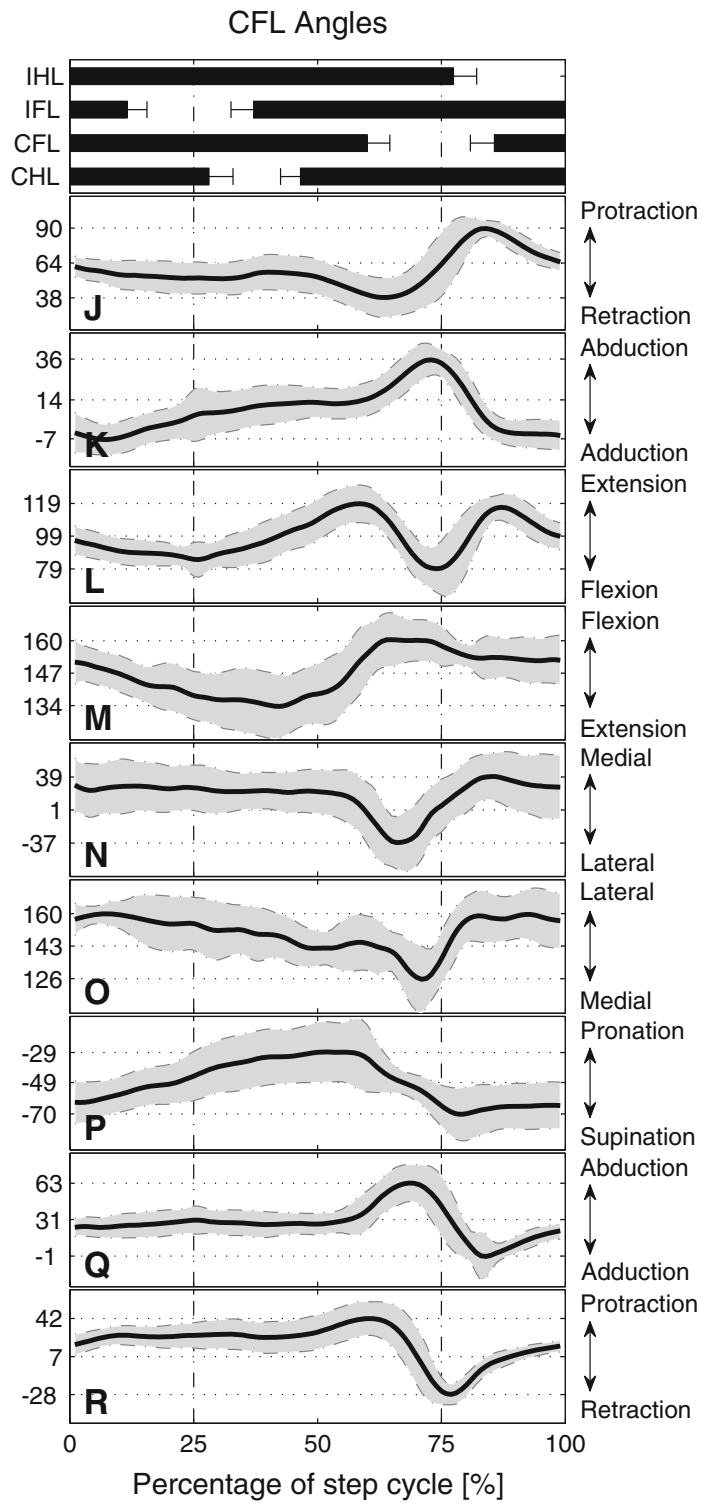

angle of the femur/humerus with zero indicating the horizontal position. $\mathbf{C}, \mathbf{L}$ Knee/elbow angle measured on flexion side. D, $\mathbf{M}$ ankle and wrist angle measured on the extension side. E, $\mathbf{N}$ Twist of the ankle/wrist (at zero twist the tarsus/carpus is in line with the crus/antebrachium). $\mathbf{F}, \mathbf{O}$ Twist (sideways deflection) of phalanges III with respect to the tarsus/carpus with $180^{\circ}$ denoting that they are in line. G, P Roll of the femur/humerus deduced from inverse kinematics of a roll-pitchyaw-knee manipulator fit onto the animal data. $\mathbf{H}, \mathbf{Q}$ Pitching of the femur/humerus, which depends on the roll angle (see Footnote 3). I, R Yaw of the femur/humerus, which produces back and forth movements when roll is zero

(Ashley-Ross 1994a, p. 256). The kinematics of the crus, tarsus, and toes do not fully support this hypothesis. In particular, quantitative results for the twist of the ankle joint (i.e., rotation of the tarsus with respect to the crus) show that the twist stops soon after the beginning of stance (20\% of step cycle; Fig. 6E). Throughout stance, rotation of the femur and pes are coupled (range of $\approx 90^{\circ}$ ). Therefore, long-axis rota- 
tion of the crus (range $\approx 30^{\circ}$, Fig. $6 \mathrm{E}$ ) only contributes $33 \%$ to the rotation of the foot. The remaining $66 \%$ results from the twist (or the horizontal rotation) of the toes with respect to the tarsus (metatarso-phalangeal twist). Indeed, the amplitude of the horizontal angle between tarsus and middle toe is $\approx 60^{\circ}$ (Fig. 6F). These two aforementioned twists do not overlap in timing. The twisting of the ankle starts with the beginning of stance and ends at $20 \%$ of the cycle. Then, there is a short period without any twist in either joint. Starting around the middle of the stance phase, the middle finger then rapidly starts twisting until the femur and knee are maximally retracted and extended, respectively.

Rolling of the hindlimbs was first hypothesized by Barclay (1946), when he proposed the "double crank" mechanism for the forelimbs of the toad. This mechanism assumes that the whole limb is only retracted by femoral rotation caused by caudofemoralis muscle activity $(\mathrm{CDF}){ }^{2}$ Later, other authors supported this idea (Gray 1968; Edwards 1977, 1989; Peters and Goslow 2010). However, Ashley-Ross listed three main reasons why this mechanism can only apply to a small portion of hindlimb retraction (Ashley-Ross 1994a, p. 256): (1) the hindlimb is almost fully extended at the beginning of the stance and thus femoral rotation would have a negligible effect on the foot's craniocaudal translation, (2) similarly, close to the end of stance, the knee is maximally extended leading to same problem as above, and (3) the CDF, as reported by Peters and Goslow (2010), is only active during the second quarter of the stride cycle. From our $\mathrm{X}$-ray analysis, the decoupling of the rolling angle of the hindlimb and the combined retraction using inverse kinematics support the points 1 and 3 . Indeed, rolling starts to have an effect only after $25 \%$ of the step cycle (Fig. 6G) while limb retraction results from the backward yaw (horizontal backward rotation) of the femur only (Fig. 6I). However, the second claim from Ashley-Ross remains questionable. Roll rapidly increases after the two-thirds of the stance phase (Fig.6G) and at this point yaw has almost stopped to contribute to femoral retraction even if retraction continues until the $\approx 60 \%$ of the step cycle. However, pitching angle ${ }^{3}$ rapidly increases in this period (Fig. $6 \mathrm{H}$ ). This suggests that the rolling of the femur combined with the downward pitch indirectly continues to supply retraction until its maximal value. That the activity of the CDF stops earlier in the stance does not mean that rolling stops. Rolling can be induced from gravity with the body pushing downward at the base of the retracted and fully extended hindlimb,

\footnotetext{
2 The CDF originates from the Crista ventralis of the femur (trochanter) close and cranioventrally to the femoral head and inserts on the anteroventral part of the tail (spanning 2-3 vertebrae).

3 The pitch angle when the roll is zero moves the femur dorsoventrally. If the roll changes to $90^{\circ}$ the pitch will be moving the femur cranioventrally.
}

while the fingers push upward to the other end. Therefore, it is reasonable to suggest that femoral retraction is active for the first two-thirds of the stance phase, while femoral rotation is for the last two-thirds.

\subsubsection{Forelimb kinematics during terrestrial stepping}

The most detailed study on the function and biomechanics of the forelimb during forward terrestrial stepping was presented by Evans (1946). Additionally, to providing insight into the forelimb's functions during normal locomotion, Evans also demonstrated their potential role for body propulsion. When the hindlimbs were disabled, bound to the sides of the body with a strip of paper, the forelimbs generated the body's propulsion, whereas in the reversed case the hindlimbs were much less effective. Moreover, salamanders vertically suspended from a glass edge could pull themselves up using their forelimbs only, illustrating the potential propulsive strength of the forelimbs (Evans 1946, p. 267). Regarding forelimb kinematics, new quantitative data were provided by Ashley-Ross et al. (2009). Detailed three-dimensional kinematics were also extracted from our cineradiographic recordings of Pleurodeles waltl.

The kinematics of the forelimbs differs fundamentally from that of the hindlimbs. Stance phase starts with the fingers touching the ground (Evans 1946; for convenience, we will refer to the movements of the forelimb contralateral to the hindlimb used as the reference limb for the stride cycle in Fig. 6). Humeral retraction begins almost synchronously with the beginning of the stance phase. At this moment, the humerus is maximally protracted (perpendicular to the scapula, Fig. 6J; Ashley-Ross et al. 2009, p.249) and maximally adducted (36 ${ }^{\circ}$, Fig. 6K). In contrast, Evans (1946, p. 264) reported maximal protraction angles close to $45^{\circ}$ (cranially from the transverse plane). During the first fifth of the forelimb's stance phase and until its contralateral hindlimb touches the ground, the humerus is rapidly retracted to almost half of the total retraction range $\left(26^{\circ}\right)$. Then, the position of the humerus remains almost constant for the next three fifths of stance (almost $50 \%$ of the step cycle) to then rapidly complete its retraction during the last fifth at the same retraction velocity as observed during the first fifth (reaching $38^{\circ}$ from the scapula; Fig. 6J; Evans 1946, p. 263). During swing phase, the elbow maximally flexes for the first half and then maximally extends during the second half (Fig. 6L). The latter contradicts Evans' observation that "the elbow is flexed all during the recovery phase". The end of the extension coincides with the touch-down of the hand. Then, the elbow slowly flexes until the spine is straight ( $25 \%$ of step cycle). While the spine bends to the side ipsilateral to the forelimb, the elbow extends and thus generates forward propulsion. A big difference between fore- and hindlimbs is the function of the distal segment. The extension of the wrist joint during stance 


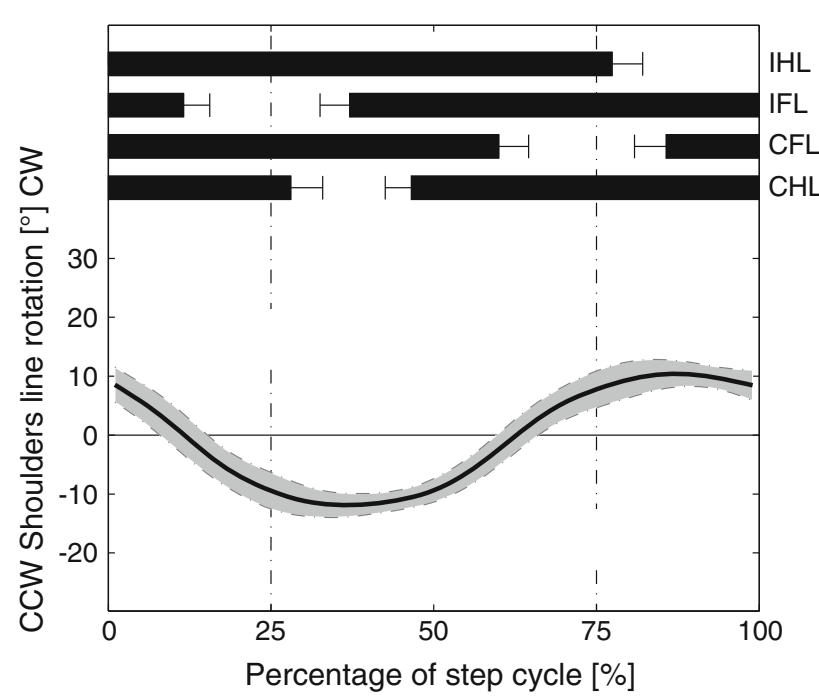

Fig. 7 Rotation of the pectoral girdle about its dorsoventral axis calculated using a line connecting the two shoulder joints and expressed relative to the line connecting the second and fourth vertebrae (region of pectoral girdle; two animals, $N=23$ total). The CW and CCW rotations follow the right-hand rule with the thumb representing the dorsoventral axis of the third vertebra and pointing dorsally

is only half the range of the ankle joint and its twist remains essentially constant (Fig. 6M, N). Therefore, the movements of the carpus with respect to the antebrachium are much more restricted than the ones of the tarsus and crus. Similarly, the fingers of the forelimb (metacarpo-phalangeal joint) twist less medially than the hindlimb's toes do (Fig. 6O). The smaller twist in the carpus is likely connected to the smaller lateral displacement of the pectoral girdle compared with the pelvic girdle (Ashley-Ross 1994a, p. 278) and because the orientation of the manus does not change as much.

Ashley-Ros et al. (2009, p. 249ff) highlighted an important difference between the functions of the limbs, specifically in the way pes and manus are placed. Their suggestion that hand placement is achieved through elbow flexion contradicts our recent cineradiographical analysis. The placement of the manus in front of the shoulder cannot be reached by humeral protraction alone as the humerus does not exceed a position perpendicular to the spine. Thus, placement of the manus is a result of the humeral roll (Fig. 6P), which brings the manus from a position behind the elbow to a position in front during swing phase. During this rotation of the antebrachium, elbow and wrist joints flex most probably for ground clearance. Afterward, forward motion of the manus is achieved by elbow extension up to $119^{\circ}$ (Fig. 6L). Additional forward motion of the manus is achieved by scapular movements. Both scapulae rotate more or less as one unit around the dorsoventral axis of the pectoral girdle. As a result, the line connecting the shoulder joints rotates symmetrically back and forth with an amplitude of $\approx 20^{\circ}$ (Fig. 7).
The analysis of the roll, pitch, and yaw movements of the humerus contralateral to the reference hindlimb (see Sect.3.1.3 for definitions) showed that humeral roll occurred almost completely throughout the hindlimb's stance phase (Fig. 6P). Contrary, humeral yaw and pitch (retractors) were observed only for a short period before and after the forelimb's swing phase (Fig. 6Q, R). Therefore, Edwards' estimations Edwards (1977) for the role of the limbs' roll to body propulsion (10-18\%) may not apply equally to the fore- and hindlimbs.

That humeral retraction is mostly inactive during the stance phase of the forelimb, humeral roll is active for about $75 \%$ of the stance phase and elbow flexion-extension range is relatively small, may suggest that the "double crank" mechanism proposed for the forelimbs of the toad from (Barclay 1946) can very well describe the movements of the salamander's forelimbs. In particular, for the $50 \%$ of the cycle, the humerus is constantly retracted at around $60^{\circ}$ from the body and rolls backward with elbow always close to $90^{\circ}-100^{\circ}$.

\subsection{Aquatic stepping}

Aquatic stepping was likely the ancestral locomotor function of the tetrapod limb (Edwards 1989; Coates and Clack 1991; Clack 2002b), which makes the study of aquatic stepping of salamanders particularly intriguing and will help to identify some of the fundamental differences that terrestrial and aquatic environments impose. However, to date, little has been described in the literature concerning aquatic stepping of salamanders.

Postural kinematics appear to be remarkably similar between the two environments for both the spine and the limbs (Ashley-Ross et al. 2009). However, some differences between aquatic and terrestrial stepping have been described. For example, averaged speed during underwater stepping is twice the speed observed during terrestrial locomotion (Ashley-Ross et al. 2009). Duty factor is greatly reduced during aquatic stepping such that the resulting footfall patterns resemble a running trot with short periods of suspension (Fig. 3 in Ashley-Ross et al. 2009; Deban and Schilling 2009). Another difference is the switch from a lateral to a diagonal sequence walk when salamanders transit from a terrestrial to an aquatic environment (Ashley-Ross 1994a). While the kinematics were found to be generally similar in the two environments, a greater variability of the locomotor parameters in their underwater recordings was noted. AshleyRoss et al. (2009, p. 251) suggested that the greater variability during aquatic stepping may be partly due to the larger vertical movements of the pelvic girdle - the structure in focus in this study.

Standing and traveling waves, the latter occurring mainly in the tail region, have been reported for underwater stepping (Lamarque et al. 2009). Although the amplitude of the 
trunk undulation was comparable between aquatic and terrestrial stepping, a small but significant (time to minimum trunk angle, $F_{1,2}=562, P=0.001$; time to maximum trunk angle, $F_{1,2}=2172, P=0.0005$, from Ashley-Ross et al. 2009) shift in the timing of maximal bending was observed (Fig. 4 in Ashley-Ross et al. 2009). The same study described that compared to terrestrial stepping, pectoral girdle rotation was greatly reduced or nonexistent during aquatic stepping, while very similar, albeit temporally shifted pelvic girdle motions were observed in both environments.

Limb kinematics was found to be similar between terrestrial and aquatic stepping in most of the investigated parameters (Ashley-Ross et al. 2009, p.252ff). Apart from the timing of the footfalls (see above), two other interesting observations were made. First, the range of motion of both the humerus and the femur was reduced during underwater walking. Both segments were held nearly perpendicular to the spine throughout the cycle (Ashley-Ross et al. 2009, p. 246ff). Second, knee and elbow joints were overall more extended during aquatic walking. A hypothesis worth mentioning is that prolongation of the swing phases of the limbs are thought to be the result of hydrodynamic constraints imposed on the acceleration of the swinging limbs (Ashley-Ross et al. 2009). Fast swing phases would demand greater forces to counteract the higher reaction forces from the viscous water.

\subsection{Swimming}

The kinematics of swimming salamanders closely resemble those of anguilliform fishes (Blight 1976; Hoff et al. 1989; Frolich and Biewener 1992; D'Août and Aerts 1997). Typically, salamanders swim at a higher frequency and speed than they step (e.g., swimming: $2.13 \pm 0.24 \mathrm{~Hz}$, stepping: $0.88 \pm 0.12 \mathrm{~Hz}$, Delvolvé et al. 1997).

Their spinal undulations correspond to a craniocaudally traveling wave, which increases in amplitude as it propagates toward the tip of the tail. The smallest lateral displacement is found around the pectoral region. In the trunk, the oscillatory amplitude increases slowly, but increases quickly in the tail to reach its maximum at the tip of the tail (Frolich and Biewener 1992; D'Août and Aerts 1997, 1999.) For swimming, only traveling waves of lateral displacement (i.e., spatial kinematics) and not of lateral flexion (i.e., angular kinematics between spinal segments) have been analyzed in the literature. Even though Roos' method (Roos 1964) would be more appropriate to characterize wave-type kinematics (see Sect. 3.1), lateral displacement during swimming may more correctly reflect the latter, because contrary to stepping, the spine directly interacts with the medium. However, caudally increasing amplitudes of spatial lateral oscillation do not mean that angular intervertebral oscillations show the same trend (i.e., depending on the bending and orientation of the spine, pronounced displacement of the tail's tip toward one side may still occur even if the caudal part of the tail bends in the opposite direction). This is particularly important when relating spinal kinematics with EMG activity.

Per swimming cycle, more than one wave travels down the spine. For example, D'Août and Aerts (1997) reported that, independent from speed, salamanders use $1.67( \pm 0.27)$ waves per body length which did not correlate significantly with swimming speed. Similarly, Frolich and Biewener (1992) reported a mean traveling wave's speed of 1.23 SVL/ cycle, which relates to 1.48 waves per body length.

\section{Kinematic studies of salamander-like locomotion in robotics}

Recently, roboticists have started using robots and numerical models to test hypotheses on how salamanders move. Additionally, new robots capable of bimodal locomotion (i.e., swimming and walking) and better controllers inspired by the neuronal locomotor circuits of salamanders have been developed. In this section, we review robotic studies related to the kinematics of salamander locomotion.

\subsection{Salamander-like robots and control}

So far, four types of gaits have been explored in robotic studies of salamander-like locomotion: (i) forward terrestrial stepping using a walking trot, (ii) terrestrial turning, (iii) forward swimming, and (iv) turning while swimming. From the short list of salamander-like robots, Salamandra robotica II (Crespi et al. 2013; Karakasiliotis and Ijspeert 2009) is the only one that addressed questions related to kinematics. Its predecessor, Salamandra robotica I (Ijspeert et al. 2007) was used mainly to demonstrate walking and swimming gaits generated by a model of the neuronal circuits which enable the transition between these two gaits.

Biomimetic robotic design Salamandra robotica II consists of nine body segments, four trunk, and three tail segments, serially connected to enable spinal movements in the horizontal plane (Fig. 8). A flexible fin at the end of the tail imitates the flattened caudal part of the animal's tail. Four limbs, attached at two of the body segments, during stepping, follow the simple "double crank" mechanism proposed by Barclay (1946) (see also Sect. 3.1.3) with only one rotational degree of freedom (DoF) that rotates the limb around an axis perpendicular to the spine. Each limb can rotate independently and the speed of rotation can be modulated throughout the locomotion cycle. Therefore, stance and swing periods can be set independently to alter the gait's pattern. During swimming, the limbs are folded backward (Crespi et al. 2013) similarly to the ones of its biological counterpart. A significant difference between the existing salamander-like robots and real salamanders for which kinematic data are available, 


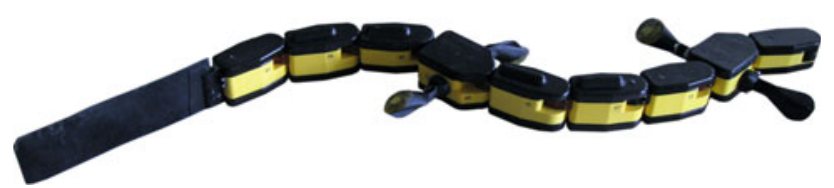

Fig. 8 The Salamandra robotica II amphibious robot

is the size. For example, Salamandra robotica robots are almost $1 \mathrm{~m}$ long and weigh around $2 \mathrm{~kg}$. Studied salamanders do not exceed lengths of $30 \mathrm{~cm}$ and masses of some tens of grams. However, some salamander species (e.g., Japanese and Chinese giant salamanders) can be up to $1.8 \mathrm{~m}$ long.

Movement generation In terms of movement generation, two types of control have been mainly used. Ijspeert et al. (2007) used a central pattern generator implemented (CPG) as a system of coupled nonlinear oscillators. A simplified version of this CPG model was used later by Crespi et al. (2013). Karakasiliotis and Ijspeert (2009) used a simple sinebased controller. Both types of control, however, use the same principles of movement generation. For the spine, each motor is driven by a sine wave of a certain amplitude, phase, and angular offset. When all sine waves are in phase (or antiphase), the robot performs a standing wave. When a phase lag is introduced between consecutive spinal segments the robot performs a traveling wave. The rotation of each limb, in both models, is driven by the phase of a sine wave. A transfer function is then used to modulate the speed of rotation for the swing and stance phases independently.

\subsection{Terrestrial stepping}

In robotics, regarding forward terrestrial salamander-like locomotion, three control parameters have been studied: (i) lateral bending in the form of intersegmental angular amplitude, (ii) locomotor frequency, and (iii) coordination between footfalls and trunk bending in the form of spine-limbs phase lag.

Lateral bending during forward walking Experiments with a kinematic model of Salamandra robotica II and the real robot showed that higher trunk bending yields greater stride length (Karakasiliotis and Ijspeert 2009; Crespi et al. 2013. However, both the model and the robot showed that there is a maximum value of bending after which the stride length starts to decay (Fig. $3 b$ in Karakasiliotis and Ijspeert 2009). To a large extent, this decelerating performance is a result of the "double crank" mechanism used for the limbs (i.e., feet positions are directly coupled to spinal kinematics). Higher body bending yields greater variability in the distance between the diagonal feet in stance (Fig. 4a in Karakasiliotis and Ijspeert 2009) which, in turn, yields greater ground slipping. Such slipping due to trunk bending is avoided in salamanders primarily through knee/ankle extension and flexion at the beginning and midway of the

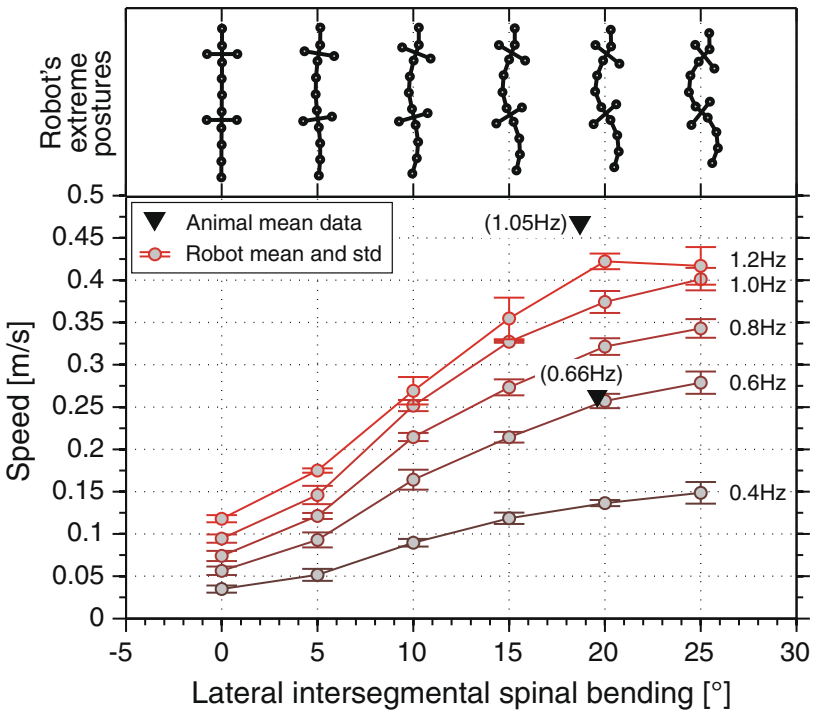

Fig. 9 Forward walking experiments with Salamandra robotica II. Each curve shows the speed of the robot for different frequencies and amplitudes of spinal bending. Top postures of the robot are shown for each value of maximal bending. Two mean values of animal data are scaled to match the dimensions of the robot and shown with black triangles (adapted from Crespi et al. 2013)

stance respectively (see Sect. 3.1.3). Real salamanders do not alter significantly the amplitude of trunk bending (Table 2 in Ashley-Ross 1994b). However, a quantitative comparison between the performances of Salamandra robotica II and real salamanders may suggest why salamanders use such a narrow range of bending amplitudes. This comparison is shown in Fig. 9. First, mean values of body bending for salamanders (data from Ashley-Ross 1994b) are near the threshold value of bending after which the robot's speed reaches a plateau for each frequency level. Moreover, Karakasiliotis and Ijspeert (2009) estimated in simulation that locomotor efficiency (as a sum of the power consumption at each joint modeled as an idealized motor) is optimal for bending angles lower than the threshold (Fig. 12 in Karakasiliotis and Ijspeert 2009). Therefore, the narrow range of bending angles in salamanders may be a result of the trade-off between stride length and energy efficiency. It is worth noting that Crespi et al. (2013) reported striking similarities in the performance and kinematics of Salamandra robotica II and real salamanders which is also evident in Figs. 9 and 10.

Further experiments with Salamandra robotica II and in simulation showed that the tail and its kinematics can significantly alter forward body propulsion, especially on slippery surfaces (Karakasiliotis and Ijspeert 2009). In particular, the robot's stride length was decreased when its tail was removed. Contrary, stride length was increased when the tail was oscillating with higher bending values (Fig. 14(a) in Karakasiliotis and Ijspeert 2009). The use of the tail as a "fifth limb" oscillating at high amplitudes was observed in salamanders when 


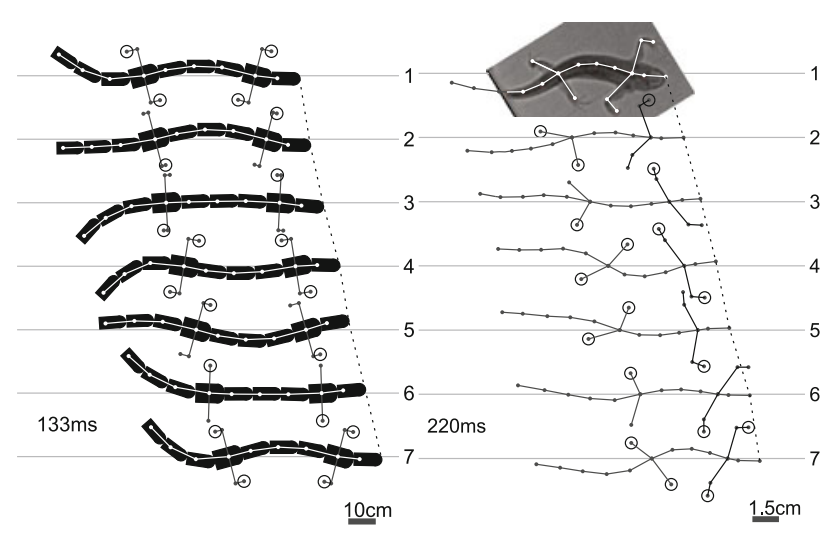

Fig. 10 Left snapshots of Salamandra robotica II during one walking cycle for the highest recorded speed. Right snapshots of a salamander during one walking cycle. The size scale is given at the bottom right of each sequence and time between each snapshot at the bottom left. The feet in stance are highlighted with a circle (adapted from Crespi et al. 2013)

walking on slippery surfaces (J. M. Cabelguen, unpublished observation).

Limbs So far, only experiments using simple rotational limbs have been done with robots. Therefore, an evaluation of Edwards' (1977) estimation concerning the contribution of limb retraction to body propulsion (see Sect. 3.1.3) is not possible yet. However, some preliminary hypotheses can be formulated based on the results from Crespi et al. (2013) (see also Fig. 9). For low locomotor frequencies, the robot's performance is similar to that of salamanders (in terms of relative speed). Because the robot lacks retraction capabilities and its body propulsion is only achieved by girdle and limb rotation, Edwards' estimation of limb retraction contributing 56-62\% to body propulsion is rather high and therefore questionable. Of course this does not address other reasons for which more complex limbs are useful (i.e., less constraints on the spine and longer retraction ranges), but rather highlights the point that limb retraction might not be as important as estimated at low locomotor frequencies. For higher frequencies, however, the robot's performance is lower than that of the salamander. As frequency increases, speed does not follow in a linear fashion as was shown for animals. The nonlinear relationship between speed and frequency might therefore be related to the lack of limb retraction.

Spine-limbs coordination In salamanders, footfalls of diagonal limbs entering stance coincide with the maximal bending of the trunk toward the ipsilateral hindlimb (Sect.3.1.3). Experiments with Salamandra robotica II showed that maximal stride length is achieved only with the above coordination (Fig. 11, a phase lag of zero indicates that the feet touch the ground in perfect synchrony with the bending of the spine). A dramatic decrease in performance was found outside a narrow range of this optimal coordination with several values leading to zero speed. Crespi et

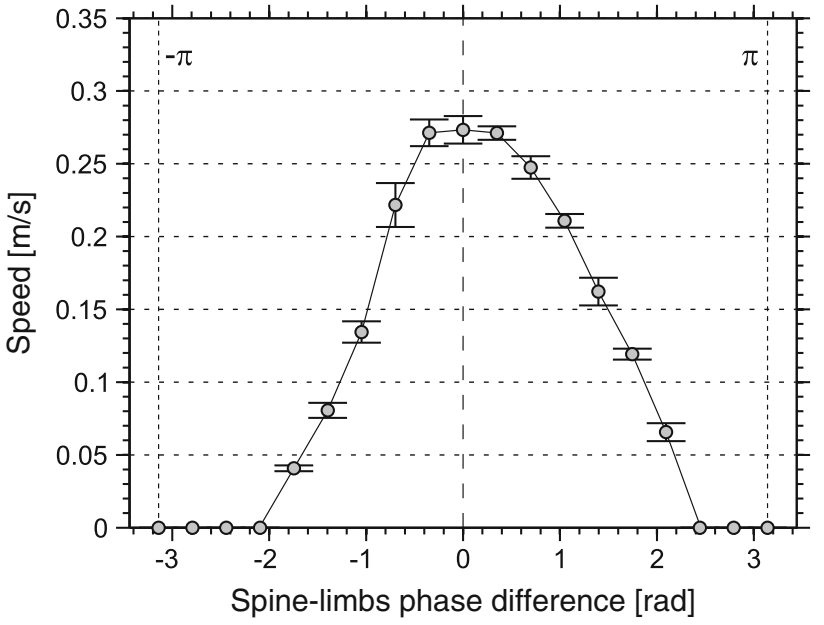

Fig. 11 Spine-limbs coordination experiment using Salamandra robotica II. The curve shows the speed of the robot with respect to the phase difference between limbs' footfall and maximal lateral bending (zero means that these two coincide; adapted from Crespi et al. 2013).

al. (2013) showed that the optimal coordination results in a greater step length as limb protraction and retraction are enhanced through girdle rotation.

Turning while walking Crespi et al. (2013) explored the effect of asymmetric spinal bending on the locomotor trajectory of Salamandra robotica II. They showed that a single offset in the oscillation of each spinal joint is capable of curving the robot's trajectory toward the offset side. Higher offset yields higher curvature, i.e., sharper turns. Harischandra et al. (2010) used a 3D musculo-mechanical model of a salamander to explore turning through bending offset, asymmetry of knee flexion-extension and their combination. In particular, they showed that both methods can change the curvature of the locomotion's trajectory and that their combination can yield even sharper turning. The latter could suggest possible ways used by salamanders to turn. However, turning has not been studied in salamanders yet and, therefore, no kinematic data are available.

\subsection{Swimming}

Forward swimming Crespi et al. (2013) conducted experiments using Salamandra robotica II to explore basic parameters of forward swimming. They used a traveling wave to actuate the robot's spine, similar to real salamanders (see Sect.3.3) and explored three main undulatory parameters: (i) amplitude, (ii) frequency, and (iii) the number of waves traveling along the spine. They found significant influence of all three parameters on swimming speed. In particular, they showed that higher undulatory amplitudes and frequencies, but lower number of waves, increased speed. A comparison of the robot's swimming stride length (see D'Août and Aerts 1997, for definition) with the one of salamanders 


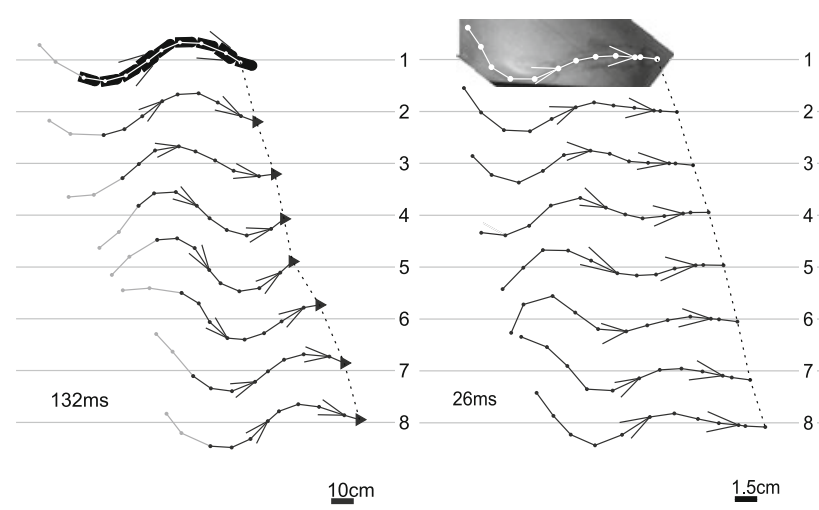

Fig. 12 Left snapshots of Salamandra robotica II during one cycle of swimming. Right snapshots of a salamander during one cycle of swimming (adapted from Crespi et al. 2013)

suggested that, during steady state swimming, salamanders account for locomotor efficiency rather than speed. The maximal stride length found with the robot was higher (0.447) than the one reported by D'Août and Aerts (1997), for salamanders $(0.345( \pm 0.077))$. However, the maximum stride length was achieved with approximately 1 wave per body length by the robot, while salamanders typically use $1.675( \pm 0.27)$ waves per body length. Similar kinematics between the robot and salamanders were also found for swimming (Crespi et al. 2013; Fig. 12). However, all swimming experiments performed in Crespi et al. (2013) used a constant curvature of lateral undulations. The main reason behind this simplification was the reduction of the parameters used for the characterization of the robot. In salamanders, the lateral undulatory curvature varies along the spine (Sect. 3.3). The result of this simplification can be seen in Fig. 12. The robot's curvature is more or less constant along the body, while the curvature increases craniocaudally in the salamander. As a result, head orientation is more variable in the robot than the animal.

Turning while swimming Turning is generated in the same way during swimming as during walking, i.e., by varying the offset of spinal undulations. (Crespi et al. 2013) explored the effect of the undulatory amplitude, offset and number of waves along the spine on the curvature of the robot's trajectory. Contrary to the terrestrial turning experiments the amplitude of spinal undulations, for non-zero values, did not show an important effect on the turning curvature. The same was reported for the number of waves. As expected, significant effect was found for the level of the undulatory offset. Higher offset values yield sharper turning, as shown also for terrestrial turning.

\section{Conclusion}

Despite the interest of several scientific disciplines on the locomotion of salamanders, quantitative data on their kine- matics are sparse and limited to a small number of species. Although in the last two decades some studies started looking into the three-dimensional kinematics, a lot remains to be done and more species need to be studied to properly address fundamental questions about the general patterns of locomotion in salamanders along with the effects of interspecific variability, speed, and environmental conditions.

Most of the studies reviewed here have shown that the lateral movements of the salamander's spine during terrestrial locomotion resemble S-shaped standing waves particularly at low speeds. However, it is as yet unclear whether traveling waves are present. This is not only a result of the small number of species examined, or the small range of speeds for which results exist, but also a result of the incompatible methods used to deduce wave types. Therefore, as a common and most appropriate tool for characterizing waves of lateral undulation, we propose to use Roos (1964) method. For swimming, spinal kinematics are much more consistent. Traveling waves of caudally increasing amplitudes and wavelengths of almost two-thirds of the animals' body length propagate along the spine.

Most of the studies presenting limb kinematics concurred with each other with only minor differences. However, some points were deduced from the detailed terrestrial kinematics of Pleurodeles waltl, which were not available for other species. First, the significant differences in the functions of fore- and hindlimbs: forelimbs' kinematics depend on the movements of the hindlimbs. In particular, their function is mostly rotational when the hindlimbs are in stance, while bursts of retraction happen when the hindlimbs are in the transition phases. Contrary to this, hindlimb kinematics show more translational (retraction-protraction) movements compared with the forelimbs. Second, the forward orientation of the pes is not only a result of crus rotation (33\%) but also of metatarso-phalangeal twist. Third, protraction and retraction of the forelimbs is enhanced by scapular rotation about the dorsoventral axis of the pectoral girdle. Many of the differences found among the various studies of limb kinematics likely result from examining different species and/or from differences in the methods used to deduce kinematic profiles, i.e., varying use of kinematic reference frames.

One way for broadening our knowledge and understanding of how salamanders move is to study detailed kinematics for several species and in different environments and environmental conditions. Although this is feasible, it is rather time consuming. Modern developments in robotics and modeling could accelerate this process. Even in early stages, studies of salamander-like locomotion with robots have shown good potential for exploring and answering biological questions. Simple robotic designs, such as the one of Salamandra robotica robots which are planar and lack retraction capabilities, have successfully addressed questions of the effect of speed on basic spinal undulatory parameters and the coordination 
between spinal and limb movements. Given that detailed kinematics for more salamander species and different environments will be provided in the future, it might be possible to develop numerical models and robotic prototypes that imitate the kinematics of real salamanders with suitable precision. Such models and robots will allow us to rapidly and systematically answer questions and generate hypotheses concerning the kinematics of different gaits and speeds of locomotion varying body morphologies and environmental effects along with specific variations of habitat properties (e.g., friction and viscosity levels).

Advancements in our understanding on how salamanders move can be advantageous for robotic design and control. Robots that incorporate morphological or control properties deduced from animal studies will likely become more agile and efficient. Adaptive and agile robotic locomotion in realistic environments is not yet solved. Salamander-like robots have a great potential toward this target, not only for their intrinsic stability but also because they may be able to perform multiple modes of locomotion.

Several things remain to be explored in robotics. So far, the majority of such studies explored only kinematics and for limited morphologies and gaits. However, several interesting questions may be explored for the link between morphology, gaits, and ecological niches that could potentially address questions related to evolutionary transitions (i.e., water to land, limb postures, etc.). Moreover, dynamics and locomotor efficiency need to be explored and be compared to animal recordings.

Acknowledgments This work was funded by the EU within the FP7 project Lampetra. Robert Salzer and Rommy Petersohn helped with the X-ray recordings and Alexander Haas and Tamer Fawzy together with Felix Beckmann from the HASYLAB at the DESY in Hamburg (Germany) prepared the scans for the 3D reconstruction of the skeleton. This study was partly supported by the Center of Interdisciplinary Prevention of Diseases related to Professional Activities funded by the Friedrich-Schiller-University Jena and the Berufsgenossenschaft Nahrungsmittel und Gastgewerbe Erfurt (Germany). All experiments were carried out in accordance with the animal welfare guidelines of the state of Thuringia, Germany (Reg.-Nr. 02-042/09). Pleurodeles walt is currently not endangered (IUCN status 2011: near threatened).

\section{References}

Ashley-Ross MA (1992) The comparative myology of the thigh and crus in the salamanders Ambystoma tigrinum and Dicamptodon tenebrosus. J Morphol 211:147-163

Ashley-Ross MA (1994a) Hind limb kinematics during terrestrial locomotion in a salamander (Dicamptodon tenebrosus). J Exp Biol 193:255-283

Ashley-Ross MA (1994b) Metamorphic and speed effects on hind limb kinematics during terrestrial locomotion in the salamander Dicamptodon tenebrosus. J Exp Biol 193:285-305

Ashley-Ross MA (1995) Patterns of hind limb motor output during walking in the salamander Dicamptodon tenebrosus, with comparisons to other tetrapods. J Comp Physiol A 177:273-285
Ashley-Ross MA, Bechtel BF (2004) Kinematics of the transition between aquatic and terrestrial locomotion in the newt Taricha torosa. J Exp Biol 207(3):461-474

Ashley-Ross MA, Lauder GV (1997) Motor patterns and kinematics during backward walking in the pacific giant salamander: evidence for novel motor output. J Neurophysiol 78(6):3047-3060

Ashley-Ross MA, Lundin R, Johnson KL (2009) Kinematics of level terrestrial and underwater walking in the california newt, Taricha torosa. J Exp Zool A 311:240-257

Azizi E, Horton JM (2004) Patterns of axial and appendicular movements during aquatic walking in the salamander Siren lacertina. Zoology 107(2):111-120

Barclay OR (1946) The mechanics of amphibian locomotion. J Exp Biol 23:177-203

Bennett AF, Garland T, Else PL (1989) Individual correlation of morphology, muscle mechanics, and locomotion in a salamander. Am J Physiol 256(6):R1200-R1208

Bennett W, Simons R, Brainerd E (2001) Twisting and bending: the functional role of salamander lateral hypaxial musculature during locomotion. J Exp Biol 204:1979-1989

Blight AR (1976) Undulatory swimming with and without waves of contraction. Nature 264(5584):352-354

Cabelguen JM, Ijspeert AJ, Lamarque S, Ryczko D (2010) Axialdynamics during locomotion in vertebrates: lesson from the salamander. Prog Brain Res 187:149-162

Carrier DR (1993) Action of the hypaxial muscles during walking and swimming in the salamander Dicamptodon ensatus. J Exp Biol 180:75-83

Carroll RL (1988) Vertebrate paleontology and evolution. W. H. Freeman \& Company, New York

Chevallier S, Ijspeert AJ, Ryczko D, Nagy F, Cabelguen JM (2008) Organisation of the spinal central pattern generators for locomotion in the salamander: biology and modelling. Brain Res Rev 57(1): $147-161$

Clack JA (2002a) An early tetrapod from “romer's gap”. Nature 418: $72-76$

Clack JA (2002b) Gaining ground: the origin and evolution of tetrapods. Indiana University Press, Bloomington

Coates MI, Clack JA (1991) Fish-like gills and breathing in the earliest known tetrapod. Nature 352:234-236

Crespi A, Karakasiliotis K, Ijspeert AJ (2013) Salamandra robotica II: an amphibious robot to study salamander-like swimming and walking gaits. Trans Robot. doi10.1109/TRO.2012.2234311

Daan S, Belterman T (1968) Lateral bending in locomotion of some lower tetrapods. Proc Ned Akad Wetten C71:245-266

D'Août K, Aerts P (1997) Kinematics and efficiency of steady swimming in adult axolotls (Ambystoma mexicanum). J Exp Biol 200(13): 1863-1871

D'Août K, Aerts S (1999) The kinematics of voluntary steady swimming of hatchling and adult axolotls (Ambystoma mexicanum shaw, 1789). Belg J Zool 129(1):305-316

Deban SM, Schilling N (2009) Activity of trunk muscles during aquatic and terrestrial locomotion in Ambystoma maculatum. J Exp Biol 212(18):2949-2959. http://zotero://attachment/457/

Delvolvé I, Bem T, Cabelguen JM (1997) Epaxial and limb muscle activity during swimming and terrestrial stepping in the adult newt, Pleurodeles waltl. J Neurophysiol 78:638-650

Edwards JL (1977) The evolution of terrestrial locomotion. In: Hecht MK, Goody PC, Hecht BM (eds) Major patterns in vertebrate evolution. Plenum, New York

Edwards JL (1989) Two perspectives on the evolution of the tetrapod limb. Am Zool 29:235-254

Evans FG (1946) The anatomy and function of the foreleg in salamander locomotion. Anat Rec 95:257-281

Francis ET (1934) The anatomy of the salamander. The Clarendon Press, Oxford 
Frolich LM, Biewener AA (1992) Kinematic and electromyographic analysis of the functional role of the body axis during terrestrial and aquatic locomotion in the salamander Ambystoma tigrinum. J Exp Biol 62:107-130

Gans C, De Gueldre G (1992) Striated muscle: physiology and functional morphology. In: Feder ME, Burggren WW (eds) Environmental physiology of the amphibians. University of Chicago Press, Chicago

Gao KQ, Shubin NH (2001) Late jurassic salamanders from northern china. Nature 410:574-577

Gray J (1944) Studies in the mechanics of the tetrapod skeleton. J Exp Biol 20(2):88-116

Gray J (1968) Animal locomotion. W. W. Norton \& Company, New York

Gregory WK, Camp CL (1918) Studies in comparative myology and osteology. Bull Am Mus Nat Hist 38:447-563

Harischandra N, Cabelguen JM, Ekeberg O (2010) A 3d musculomechanical model of the salamander for the study of different gaits and modes of locomotion. Front Neurorobot 4:112

Hildebrand M (1966) Analysis of the symmetrical gaits of tetrapods. Fol Biotheor 6:9-22

Hildebrand M (1976) Analysis of the symmetrical gaits of tetrapods. In: Herman RM, Grillner RMS, Stein PSG, Stuart DG (eds) Neural control of locomotion. Plenum, New York, pp 203-236

Hildebrand M (1985) Walking and running. In: Hildebrand M, Bramble DM, Liem KF, Wake DB (eds) Functional vertebrate morphology. Belknap Press, Cambridge, pp 28-57

Hoff KV, Huq N, King VA, Wassersug RJ (1989) The kinematics of larval salamander swimming (ambystomatidae: Caudata). Can J Zool 67(11):2756-2761

Howell AB (1944) Speed in animals. Hafner Publishing Company, New York

Ijspeert AJ (2008) Central pattern generators for locomotion control in animals and robots: a review. Neural Netw 21(4):642-653

Ijspeert A, Crespi A, Ryczko D, Cabelguen JM (2007) From swimming to walking with a salamander robot driven by a spinal cord model. Science 315(5817):1416-1420
Karakasiliotis K, Ijspeert AJ (2009) Analysis of the terrestrial locomotion of a salamander robot. In: Intelligent robots and systems, pp $5015-5020$

Lamarque S, Ryczko D, Didier H, Cabelguen JM (2009) Dynamics of the axial locomotor network in intact, freely moving salamanders. In: 31 st International symposium GRSNC: breathe, walk and chew-the neural challenge (Abstract 22)

Niedzwiedzki G, Szrek P, Narkiewicz K, Narkiewicz M, Ahlberg PE (2010) Tetrapod trackways from the early middle devonian period of poland. Nature 463:43-48

Peters SE, Goslow GE (2010) From salamanders to mammals: continuity in musculoskeletal function during locomotion. Brain Behav Evol 22:191-197

Rewcastle SC (1981) Stance ang gait in tetrapods: an evolutionary scenario. Symp Zool Soc Lond 48:239-267

Romer AS (1922) The locomotor apparatus of certain primitive and mammal-like reptiles. Bull Am Mus Nat Hist 46:517-606

Romer AS, Byrne F (1931) The pes of Diadectes: notes on the primitive tetrapod limb. Palaeobiologica 4:25-48

Roos PJ (1964) Lateral bending in newt locomotion. Proc Ned Akad Wetten C67:223-232

Schaeffer B (1941) The morphological and functional evolution of the tarsus in amphibians and reptiles. Bull Am Mus Nat Hist 78:395-472

Sheffield KM, Blob RW (2011) Loading mechanics of the femur in tiger salamanders (Ambystoma tigrinum) during terrestrial locomotion. J Exp Biol 214:2603-2615

Simons RS, Brainerd EL (1999) Morphological variation of hypaxial musculature in salamanders (lissamphibia: Caudata). J Morphol 241(2):153-164

Székely G, Czéh G, Vörös G (1969) The activity pattern of limb muscles in freely moving normal and deafferented newts. Exp Brain Res 9(1):53-62

Worthington RD, Wake DB (1972) Patterns of regional variation in the vertebral column of terrestrial salamanders. J Morphol 137(3): 257-277 